\title{
Addendum: Kea show three signatures of domain-general statistical inference
}

\author{
Amalia P. M. Bastos (1) \& Alex H. Taylor (1)
}

Addendum to: Nature Communications https://doi.org/10.1038/s41467-020-14695-1, published online 3 March 2020.

In the original version of the Article, our experimental and data processing methods did not account for the fact that tokens may have been visible between the experimenter's fingers in some of the experimental trials. This could have provided kea with additional information when selecting between the jars in the experiment.

To rectify this issue, three independent coders first reviewed the video data files for all trials $(n=720)$ to identify the trials in which both experimenters' hands were visible in the recording $(n=432)$. The video files for these trials were then reviewed using frame-byframe analysis to identify trials in which token colours were visible to the kea. Coders identified 10 potentially problematic trials, out of the total 432 coded trials, where the subject may have had additional information prior to making their choice. These trials were then either substituted with the next available training trial (for kea that did not reach the 17/20 criteria in their first 20 trials), or removed entirely where no subsequent trials were available (for kea that did reach the $17 / 20$ criteria in their first 20 trials). In the video data for the remaining 288 trials it was not possible to see both experimenters' hands from an appropriate angle. Therefore, we randomly applied the observed error rate (10/432 trials, $2.31 \%)$ to these 288 trials, and so randomly allocated 7 trials out of these 288 to be either substituted by the next available trial within that condition, or removed entirely where subsequent trials were not available. The updated version of the original Table 1 is presented below as Table 1, which summarises our results following these changes.

To ensure that these changes did not affect the overall results of our study, we have re-run all statistical analyses affected by these changes. The new Bayesian binomial tests for individual performances are now provided (Table 1). The recoding and error simulation did not significantly affect the performance of any kea in any condition over the course of our three experiments. Our intercept-only model on first trial data reveals that the likelihood of a randomly selected kea succeeding in their first trial of any condition was 0.68 ( $\mathrm{pMCMC}=0.009)$, compared to a likelihood of $0.70(\mathrm{pMCMC}=0.005)$ in the original analysis. In this updated dataset, subjects made the correct choice in $69.44 \%$ of all first trials. Considering only instances where the subject succeeded in that condition, performance was correct in $77.78 \%$ of first trials. As in the original results, re-analysis of the correlation between condition number and average performance within the first 20 trials of each condition did not reveal any evidence of learning effects $(r=0.151, B F=0.509)$.

To check that our initial simulation was not an outlier, and so represents a likely set of substitutions or removals of seven randomlyassigned trials, we ran it an additional five times. In four of five of these repetitions, trial removals or substitutions did not affect subjects' performance in any blocks: above chance performances remained above chance, and chance performances remained at chance. In one of the five repetitions, the simulation affected three blocks: one showed a performance change from chance levels to above chance, and two changed from above chance performance to chance. Following the changes, Blofeld showed above performance at 15/20 in Experiment 1's Condition 2, and Plankton performed at 14/20 in the same condition, which, therefore, resulted in no difference to the total number of kea passing this test (4 of 6), and thus does not change our conclusions in any way. One of Neo's correct trials in Experiment 3 was removed without substitution, reducing his score from $15 / 20(\mathrm{BF}=3.22)$ to $14 / 19(\mathrm{BF}=2.254)$ resulting in two, rather than three kea passing this condition. Thus only in one of the six simulations that we ran in total (one for our actual results, a further five to check the simulation robustness) did performance significantly change for any kea in any condition. Furthermore, even the one significant change we did observe across our six simulations does not affect our conclusions about kea intelligence, because a further two kea passed the condition in question.

In order to ensure that no other potential biases may have affected our data, we have run a new control experiment which addresses whether kea may have relied on any unintentional experimenter cues, such as body posture or breathing patterns, to select the hand holding the rewarding black token. We presented all six individuals included in the original study with twenty trials where both jars contained 55 rewarding tokens and 55 unrewarding tokens (50:50 ratio). In each set of trials, the experimenter consistently sampled a rewarding token from one jar, and an unrewarding token from the other. As in all test conditions, the experimenters wore mirrored 


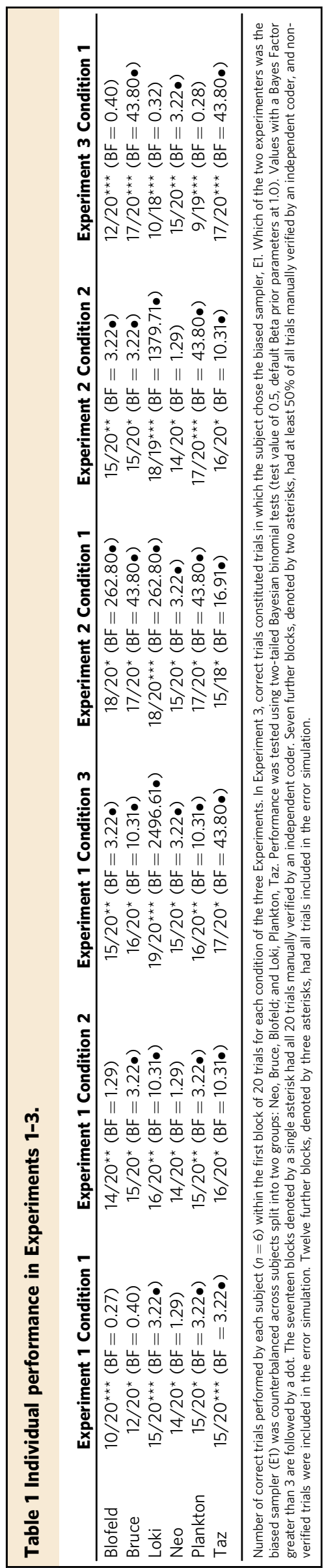

Table 2 Individual performance in experiment controlling for unintentional experimenter cues or biases.

Performance in new control Experiment

\begin{tabular}{lr}
\hline Blofeld & $12 / 20(B F=0.396)$ \\
Bruce & $13 / 20(B F=0.644)$ \\
Loki & $9 / 20(B F=0.297)$ \\
Neo & $8 / 20(B F=0.396)$ \\
Plankton & $12 / 20(B F=0.396)$ \\
Taz & $11 / 20(B F=0.297)$ \\
\hline
\end{tabular}

Number of correct trials performed by each subject $(n=6)$ within the first block of 20 trials for the experiment controlling for experimenter cues or biases. Performance was tested using twotailed Bayesian binomial tests (test value of 0.5 , default Beta prior parameters at 1.0). Values with a Bayes Factor lower than 0.3 provide strong support for the null hypothesis of no

difference from chance.

sunglasses whilst sampling from the two jars. If subjects could use any unintentional experimenter cues or biases to choose the hand containing a rewarding black token, we would expect them to perform above chance across the twenty trials in this condition. On the other hand, if subjects were relying on the proportions of rewarding and unrewarding tokens to make their choices, they should perform at chance. None of the six kea performed above chance level at this control (Table 2).

Our updated results, alongside our new control condition, show the findings and conclusions of our original article are correct. Recoding and our initial error simulation did not significantly affect the performance of any kea for any condition of the original experiments, nor the results of our other analyses, while the simulation repetitions we ran show our initial simulation run was not an outlier. Finally, the additional control condition we report here finds no support for the hypothesis that the kea used any unintentional experimenter cues to guide their choices.

\section{Acknowledgements}

We thank Jonathan Webb for his help collecting additional data for this study; and Jonathan Webb, Kata Horváth, and Patrick Wood for blind-coding our video data.

\footnotetext{
cc) (i) Open Access This article is licensed under a Creative Commons Attribution 4.0 International License, which permits use, sharing, adaptation, distribution and reproduction in any medium or format, as long as you give appropriate credit to the original author(s) and the source, provide a link to the Creative Commons license, and indicate if changes were made. The images or other third party material in this article are included in the article's Creative Commons license, unless indicated otherwise in a credit line to the material. If material is not included in the article's Creative Commons license and your intended use is not permitted by statutory regulation or exceeds the permitted use, you will need to obtain permission directly from the copyright holder. To view a copy of this license, visit http://creativecommons.org/ licenses/by/4.0/.
}

(c) The Author(s) 2020 